\title{
Mechanisms of Neutrinoless Double-Beta Decay: A Comparative Analysis of Several Nuclei
}

\author{
A. Ali ${ }^{1)}$, A. V. Borisov ${ }^{2}{ }^{*}$, and D. V. Zhuridov ${ }^{3)}$ \\ Received February 10, 2010
}

\begin{abstract}
The neutrinoless double beta decay of several nuclei that are of interest from the experimental point of view $\left({ }^{76} \mathrm{Ge},{ }^{82} \mathrm{Se},{ }^{100} \mathrm{Mo},{ }^{130} \mathrm{Te}\right.$, and $\left.{ }^{136} \mathrm{Xe}\right)$ is investigated on the basis of a general Lorentzinvariant effective Lagrangian describing physics effects beyond the Standard Model. The half-lives and angular-correlation coefficients for electrons are calculated for various decay mechanisms associated, in particular, with the exchange of Majorana neutrinos, supersymmetric particles (with $R$-parity violation), leptoquarks, and right-handed $W_{R}$ bosons. The effect of theoretical uncertainties in the values of relevant nuclear matrix elements on decay features is considered.
\end{abstract}

DOI: $10.1134 / \mathrm{S} 1063778810120136$

\section{INTRODUCTION}

The majority of Standard Model extensions aimed at explaining the observed smallness of neutrino masses predict the Majorana nature of the neutrinos. Such theories include those employing the seesaw mechanism [1] and models of neutrino-mass generation by radiative corrections $[2,3]$. An experimental observation of neutrinoless double-beta decay $(0 \nu 2 \beta)$ would be a decisive argument in favor of the Majorana nature of the neutrino, as contrasted against the Dirac nature of all of the remaining known fermions (for an overview of experiments performed now and planned for the future to seek such decays, see [4]).

We recall that $0 \nu 2 \beta$ decay is forbidden in the Standard Model by the law of lepton-number conservation. However, the Lagrangians of various Standard Model extensions may include terms that lead to lepton-number nonconservation and, hence, to the decay in question. Possible mechanisms of leptonnumber violation may involve the exchange of Majorana neutrinos [5-7], supersymmetric Majorana particles [8-13], scalar bilinears [14] (for example, doubly charged Higgs particles [3]), leptoquarks [15], right-handed bosons $W_{R}[6,7,16]$, and so on. It is necessary to separate the aforementioned different contributions to the decay process in order to extract information about the properties of lepton-numberviolation sources-in particular, about the masses

\footnotetext{
${ }^{1)}$ Deutsches Elektronen-Synchrotron, DESY, 22607 Hamburg, Germany.

${ }^{2)}$ Moscow State University, Moscow, 119991 Russia.

${ }^{3)}$ Scuola Normale Superiore, piazza dei Cavalieri 7, I-56126 Pisa, Italy.

*E-mail: borisov@phys.msu.ru
}

of (Majorana) neutrinos and the parameters of their mixing. On this path, measurements of the properties of the $0 \nu 2 \beta$ decay of various nuclei and their comparative analysis will be helpful in pinpointing the main decay mechanism [17-19]. It should be noted that the possibility of identifying different decay mechanisms was first demonstrated in [6, 7]. It was shown in [20] that the calculated ratios of nuclear half-lives are highly sensitive to the choice of model for calculating relevant nuclear matrix elements, and it was proposed there on this basis to test nuclear models by comparing these ratios with (future) experimental data on $0 \nu 2 \beta$ decay of several nuclei.

In our previous studies [21, 22], we investigated the effect of various mechanisms of the neutrinoless double-beta $(0 \nu 2 \beta)$ decay of the ${ }^{76} \mathrm{Ge}$ nucleus on the angular correlation of final-state electrons. In the present article, we generalize the results of those studies by performing a comparative analysis of the half-lives and electron angular-correlation coefficients for the ${ }^{76} \mathrm{Ge},{ }^{82} \mathrm{Se},{ }^{100} \mathrm{Mo},{ }^{130} \mathrm{Te}$, and ${ }^{136} \mathrm{Xe}$ nuclei.

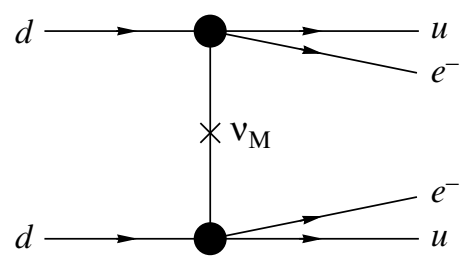

Fig. 1. Long-range mechanism of $0 \nu 2 \beta$ decay. 
Table 1. Nonzero coefficients $\epsilon_{\alpha}^{\beta}$ for various models

\begin{tabular}{l|l}
\hline \multicolumn{1}{c|}{ Model } & Nonzero $\epsilon_{\alpha}^{\beta}$ \\
\hline RC [7, 16] & $\epsilon_{V \mp A}^{V \mp A}$ \\
RPV SUSY [12] & $\epsilon_{S+P}^{S \mp P}, \epsilon_{V-A}^{V-A}, \epsilon_{T_{R}}^{T_{R}}$ \\
LQ [15] & $\epsilon_{S \mp P}^{S+P}, \epsilon_{V \mp A}^{V+A}$ \\
\hline
\end{tabular}

\section{COMPARATIVE ANALYSIS OF DECAY} MECHANISMS FOR SEVERAL NUCLEI

Following [22], we restrict ourselves to the class of long-range mechanisms of $0 \nu 2 \beta$ decay, which involve the exchange of light Majorana neutrinos $\nu_{M}$. A general structure of the corresponding Feynman diagrams is shown in Fig. 1, where four-fermion vertices are described by a general effective low-energy Lagrangian including a complete set of Lorentzinvariant terms:

$$
\begin{gathered}
\mathcal{L}=\frac{G_{\mathrm{F}} V_{u d}}{\sqrt{2}}\left[\left(U_{e i}+\epsilon_{V-A, i}^{V-A}\right) j_{V-A}^{\mu i} J_{V-A, \mu}^{+}\right. \\
\left.+\sum_{\alpha, \beta}{ }^{\prime} \epsilon_{\alpha i}^{\beta} j_{\beta}^{i} J_{\alpha}^{+}+\text {H.c. }\right] .
\end{gathered}
$$

Here, $J_{\alpha}^{+}=\bar{u} O_{\alpha} d$ are quark currents; $j_{\beta}^{i}=\bar{e} O_{\beta} \nu_{i}$ are lepton currents, where the fields $\nu_{i}$ correspond to light-mass eigenstates of Majorana neutrinos; summation is performed over the dummy indices $\alpha, \beta=$ $V \mp A, S \mp P, T_{L, R}\left(O_{T_{\rho}}=2 \sigma^{\mu \nu} P_{\rho}, \sigma^{\mu \nu}=\frac{i}{2}\left[\gamma^{\mu}, \gamma^{\nu}\right]\right.$, $P_{\rho}=\left(1 \mp \gamma_{5}\right) / 2$ is the chiral projection operator, and $\rho=L, R)$; the prime means that the term for which $\alpha=\beta=V-A$ is excluded from the sum; $U_{e i}$ stand for lepton-mixing-matrix elements [23]; and $V_{u d}$ is a quark-mixing-matrix element [24]. The coefficients $\epsilon_{\alpha i}^{\beta}$ parametrize new-physics effects [that is, the deviation of the Lagrangian from the Standard Model form of the product of currents of the $(V-A) \otimes(V-$ $A)$ type and mixing with non-SM neutrinos]. The nonzero coefficients

$$
\epsilon_{\alpha}^{\beta}=U_{e i} \epsilon_{\alpha i}^{\beta}
$$

are presented in Table 1 for a number of versions of the Standard Model extension: $\nu_{M}$ and RPV SUSY (supersymmetry with $R$-parity violation) [12] and $\nu_{M}$ and right-handed currents (RC) associated with righthanded $W_{R}$ bosons $[6,7,16]$ or leptoquarks (LQ) [15].

The differential decay width for the $0^{+}(A, Z) \rightarrow$ $0^{+}(A, Z+2) e^{-} e^{-}$transitions has the form [22]

$$
\frac{d \Gamma}{d \cos \theta}=\frac{\ln 2}{2}\left|M_{\mathrm{GT}}\right|^{2} \mathcal{A}(1-K \cos \theta),
$$

where $\theta$ is the angle between the electron momenta in the rest frame of the primary nucleus; $M_{\mathrm{GT}}$ is the
Gamow-Teller nuclear matrix element; and $K$ is the angular-correlation coefficient,

$$
K=\mathcal{B} / \mathcal{A}, \quad-1<K<1 .
$$

Expression (3) was obtained with allowance for the leading contributions of the small parameters $\epsilon_{\alpha}^{\beta}$ in (2) to the amplitude of the process - that is, either both effective four-fermion vertices in Fig. 1 have the standard form $(V-A) \otimes(V-A)$ owing to the exchange of the $W_{L}$ boson in each of them or one of the vertices is standard, while the other is a non-SM one of the $\alpha \otimes \beta$ type (with exception of $\alpha=\beta=V-A$ case) because of the exchange of a $W_{R}$ boson, a leptoquark, a superparticle, etc. The expressions for the quantities $\mathcal{A}$ and $\mathcal{B}$ appearing in (4) are given in Table 2 for various choices of only one nonzero parameter $\epsilon_{\alpha}^{\beta}$ (the case of several nonzero parameters is difficult for analysis). In the case of the standard decay mechanism (all $\epsilon_{\alpha}^{\beta}=0$ ), we have

$$
\mathcal{A}_{0}=C_{1}|\mu|^{2}, \quad \mathcal{B}_{0}=D_{1}|\mu|^{2} .
$$

Here, $\mu$ is the ratio of the (standard) effective Majorana mass $\langle m\rangle=\sum_{i} U_{e i}^{2} m_{i}$ to the electron mass $m_{e}$; the expressions for the non-SM (normalized to $m_{e}$ ) effective masses $\mu_{\alpha}^{\beta}$ and the quantities $C_{i}$ and $D_{i}$, which are different quadratic combinations of nuclear matrix elements whose coefficients are kinematical (phase-space) integrals, can be found in [22] \{where, in particular, the expressions for the nuclear parameters $\chi_{2 \pm}$ from [7] were corrected: compare $(3 \cdot 5 \cdot 16)$ therein with (49) in [22]\}; and $s_{i}=\sin \psi_{i}$ and $c_{i}=$ $\cos \psi_{i}, \psi_{i}$ being the phase differences between the contributions of the standard and non-SM decay mechanisms. It should be noted that the effect of (pseudo)scalar $(S+P)$ and tensor $\left(T_{L, R}\right)$ currents was calculated first in [22], but an independent verification is needed.

The coefficients $C_{i}$ and $D_{i}$ at $i=1,0,1+, 2,3,4$, and 5 for the decays ${ }^{76} \mathrm{Ge} \rightarrow{ }^{76} \mathrm{Se},{ }^{82} \mathrm{Se} \rightarrow{ }^{82} \mathrm{Kr}$, ${ }^{100} \mathrm{Mo} \rightarrow{ }^{100} \mathrm{Ru},{ }^{130} \mathrm{Te} \rightarrow{ }^{130} \mathrm{Xe}$, and ${ }^{136} \mathrm{Xe} \rightarrow{ }^{136} \mathrm{Ba}$ (final-state electrons are not indicated for the sake of brevity) were found with allowance for the numerical values of the corresponding nuclear matrix elements within two nuclear models: the quasiparticle randomphase approximation (QRPA) without and with allowance for $p n$ pairing [25]. These coefficients are given in Tables 3 and 4 . In our calculations, we used the values of the energy release $Q$ in $0 \nu 2 \beta$ decays from [26] and the radius of the nucleus of mass number $A$ in the form $R=r_{0} A^{1 / 3}$, where $r_{0}=1.1 \mathrm{fm}$ is the scale factor ( $R$ appears in the expressions for the kinematical integrals and nuclear matrix elements).

Not only does the use of data for various decaying nuclei make it possible to revisit three particular cases already studied in [22], 
Table 2. Quantities factors $\mathcal{A}$ and $\mathcal{B}$ in (3) and (4) for specifically chosen $\epsilon_{\alpha}^{\beta}$

\begin{tabular}{c|l|l}
\hline$\epsilon$ & \multicolumn{1}{|c|}{$\mathcal{A}$} & \multicolumn{1}{c}{$\mathcal{B}$} \\
\hline$\epsilon_{V-A}^{V-A}$ & $\mathcal{A}_{0}+4 C_{1}|\mu|\left|\mu_{V-A}^{V-A}\right| c_{02}+4 C_{1}\left|\mu_{V-A}^{V-A}\right|^{2}$ & $\mathcal{B}_{0}+4 D_{1}|\mu|\left|\mu_{V-A}^{V-A}\right| c_{02}+4 D_{1}\left|\mu_{V-A}^{V-A}\right|^{2}$ \\
$\epsilon_{V+A}^{V-A}$ & $\mathcal{A}_{0}+4 C_{0}|\mu|\left|\mu_{V+A}^{V-A}\right| c_{01}+4 C_{1+}\left|\mu_{V+A}^{V-A}\right|^{2}$ & $\mathcal{B}_{0}+4 D_{0}|\mu|\left|\mu_{V+A}^{V-A}\right| c_{01}+4 D_{1+}\left|\mu_{V+A}^{V-A}\right|^{2}$ \\
$\epsilon_{V-A}^{V+A}$ & $\mathcal{A}_{0}+C_{3}|\mu|\left|\epsilon_{V-A}^{V+A}\right| c_{2}+C_{5}\left|\epsilon_{V-A}^{V+A}\right|^{2}$ & $\mathcal{B}_{0}+|\mu|\left|\epsilon_{V-A}^{V+A}\right|\left(D_{3} c_{2}+D_{3-} s_{2}\right)+D_{5}\left|\epsilon_{V-A}^{V+A}\right|^{2}$ \\
$\epsilon_{V+A}^{V+A}$ & $\mathcal{A}_{0}+C_{2}|\mu|\left|\epsilon_{V+A}^{V+A}\right| c_{1}+C_{4}\left|\epsilon_{V+A}^{V+A}\right|^{2}$ & $\mathcal{B}_{0}+|\mu|\left|\epsilon_{V+A}^{V+A}\right|\left(D_{2} c_{1}+D_{2-} s_{1}\right)+D_{4}\left|\epsilon_{V+A}^{V+A}\right|^{2}$ \\
$\epsilon_{S-P}^{S-P}$ & $\mathcal{A}_{0}+4 C_{0}^{S P}|\mu|\left|\mu_{S-P}^{S-P}\right| c_{04}+4 C_{1}^{S P}\left|\mu_{S-P}^{S-P}\right|^{2}$ & $\mathcal{B}_{0}+4 D_{0-}^{S P}|\mu|\left|\mu_{S-P}^{S-P}\right| s_{04}+4 D_{1}^{S P}\left|\mu_{S-P}^{S-P}\right|^{2}$ \\
$\epsilon_{S+P}^{S-P}$ & $\mathcal{A}_{0}+4 C_{0}^{S P}|\mu|\left|\mu_{S+P}^{S-P}\right| c_{03}+4 C_{1}^{S P}\left|\mu_{S+P}^{S-P}\right|^{2}$ & $\mathcal{B}_{0}+4 D_{0-}^{S P}|\mu|\left|\mu_{S+P}^{S-P}\right| s_{03}+4 D_{1}^{S P}\left|\mu_{S+P}^{S-P}\right|^{2}$ \\
$\epsilon_{S-P}^{S+P}$ & $\mathcal{A}_{0}+C_{2}^{S P}|\mu|\left|\epsilon_{S-P}^{S+P}\right| c_{4}+C_{3}^{S P}\left|\epsilon_{S-P}^{S+P}\right|^{2}$ & $\mathcal{B}_{0}+|\mu|\left|\epsilon_{S-P}^{S+P}\right|\left(D_{2}^{S P} c_{4}+D_{2-}^{S P} s_{4}\right)+D_{3}^{S P}\left|\epsilon_{S-P}^{S+P}\right|^{2}$ \\
$\epsilon_{S+P}^{S+P}$ & $\mathcal{A}_{0}+C_{2}^{S P}|\mu|\left|\epsilon_{S+P}^{S+P}\right| c_{3}+C_{3}^{S P}\left|\epsilon_{S+P}^{S+P}\right|^{2}$ & $\mathcal{B}_{0}+|\mu|\left|\epsilon_{S+P}^{S+P}\right|\left(D_{2}^{S P} c_{3}+D_{2-}^{S P} s_{3}\right)+D_{3}^{S P}\left|\epsilon_{S+P}^{S+P}\right|^{2}$ \\
$\epsilon_{T_{L}}^{T_{L}}$ & $\mathcal{A}_{0}+4 C_{0}^{T}|\mu|\left|\mu_{T_{L}}^{T_{L}}\right| c_{06}+4 C_{1}^{T}\left|\mu_{T_{L}}^{T_{L}}\right|^{2}$ & $\mathcal{B}_{0}+4 D_{0-}^{T}|\mu|\left|\mu_{T_{L}}^{T_{L}}\right| s_{06}+4 D_{1}^{T}\left|\mu_{T_{L}}^{T_{L}}\right|^{2}$ \\
\hline$\epsilon_{T_{R}}^{T_{L}}, \epsilon_{T_{L}}^{T_{R}}$ & \multicolumn{2}{c}{$\mathcal{A}_{0}$} \\
\hline$\epsilon_{T_{R}}^{T_{R}}$ & $\mathcal{A}_{0}+C_{2}^{T}|\mu|\left|\epsilon_{T_{R}}^{T_{R}}\right| c_{5}+C_{3}^{T}\left|\epsilon_{T_{R}}^{T_{R}}\right|^{2}$ & $\mathcal{B}_{0}$ \\
\hline
\end{tabular}

Table 3. Numerical values of $C_{i}$ and $D_{i}$ multiplied by $10^{15}$ for various nuclei within the QRPA model without $p n$ pairing [25]

\begin{tabular}{l|c|c|c|c|c|c|c}
\hline Nucleus & $C_{1}$ & $C_{0}$ & $C_{1+}$ & $C_{2}$ & $C_{3}$ & $C_{4}$ & $C_{5}$ \\
\hline${ }^{76} \mathrm{Ge}$ & 7.208 & -6.680 & 6.191 & -4.601 & 1520 & 11.54 & $3.152 \times 10^{5}$ \\
${ }^{82} \mathrm{Se}$ & 29.97 & -29.50 & 29.03 & -15.45 & 2297 & 81.56 & $2.627 \times 10^{5}$ \\
${ }^{100} \mathrm{Mo}$ & 151.3 & -20.04 & 2.656 & -46.82 & $2.446 \times 10^{4}$ & 245.1 & $6.426 \times 10^{6}$ \\
${ }^{130} \mathrm{Te}$ & 46.67 & -46.30 & 45.93 & -35.89 & 9670 & 115.7 & $2.480 \times 10^{6}$ \\
${ }^{136} \mathrm{Xe}$ & 45.54 & -48.16 & 50.94 & -37.88 & 9896 & 112.0 & $2.581 \times 10^{6}$ \\
\hline Nucleus & $D_{1}$ & $D_{0}$ & $D_{1+}$ & $D_{2}$ & $D_{3}$ & $D_{4}$ & $D_{5}$ \\
\hline${ }^{76} \mathrm{Ge}$ & 5.872 & -5.442 & 5.044 & -0.6179 & -17.31 & -9.073 & $-2.309 \times 10^{5}$ \\
${ }^{82} \mathrm{Se}$ & 26.42 & -26.00 & 25.59 & 3.292 & -3.428 & -72.72 & $-2.154 \times 10^{5}$ \\
${ }^{100} \mathrm{Mo}$ & 133.5 & -17.69 & 2.344 & 26.62 & -773.7 & -219.1 & $-5.390 \times 10^{6}$ \\
${ }^{130} \mathrm{Te}$ & 39.62 & -39.30 & 38.99 & -5.405 & -152.7 & -92.26 & $-1.951 \times 10^{6}$ \\
${ }^{136} \mathrm{Xe}$ & 38.45 & -40.67 & 43.01 & -7.952 & -171.7 & -86.66 & $-2.014 \times 10^{6}$ \\
\hline
\end{tabular}

(A) all $\epsilon_{\alpha}^{\beta}=0,|\langle m\rangle| \neq 0(\mathrm{~S} M+$ Majorana neutrinos);

(B) $\epsilon_{\alpha}^{\beta} \neq 0, \quad|\langle m\rangle|=0$ (zero effective Majorana mass);

(C) $\epsilon_{\alpha}^{\beta} \neq 0,|\langle m\rangle| \neq 0, c_{i} \equiv \cos \psi_{i}=0$,

but this also permits investigating a more general situation,

(D) $\epsilon_{\alpha}^{\beta} \neq 0,|\langle m\rangle| \neq 0, c_{i} \neq 0$.

We restrict ourselves to analyzing the contributions of the parameters $\epsilon_{V \mp A}^{V \mp A}$ because the necessary extended [with respect to case $(A)$ ] sets of nuclear matrix elements were calculated in [25] only for these parameters. As far as we know, there are no numerical values of non-SM nuclear matrix elements (for their definition, see [22]) in more recent studies (we are grateful to F. Šimkovic for a discussion on this issue).

(A) All $\epsilon_{\boldsymbol{\alpha}}^{\boldsymbol{\beta}}=\mathbf{0},|\langle m\rangle| \neq \mathbf{0}$. Using formulas (3)(5) and the results from [22], we obtain the half-life and the angular-correlation coefficient in the form

$$
T_{1 / 2}=\left(\left|M_{\mathrm{GT}}\right|^{2} C_{1}|\mu|^{2}\right)^{-1},
$$


Table 4. Numerical values of $C_{i}$ and $D_{i}$ multiplied by $10^{15}$ for various nuclei within the QRPA model with allowance for pn pairing [25]

\begin{tabular}{l|c|c|c|c|c|c|c}
\hline Nucleus & $C_{1}$ & $C_{0}$ & $C_{1+}$ & $C_{2}$ & $C_{3}$ & $C_{4}$ & $C_{5}$ \\
\hline${ }^{76} \mathrm{Ge}$ & 3.526 & -6.188 & 10.86 & -0.745 & 1065 & 3.037 & $3.153 \times 10^{5}$ \\
${ }^{82} \mathrm{Se}$ & 59.15 & -24.39 & 10.06 & -54.58 & 3206 & 226.1 & $2.627 \times 10^{5}$ \\
${ }^{100} \mathrm{Mo}$ & 0.1805 & -5.737 & 182.3 & -1.530 & 860.9 & 37.98 & $6.428 \times 10^{6}$ \\
${ }^{130} \mathrm{Te}$ & 30.83 & -44.73 & 64.91 & -25.58 & 7878 & 80.66 & $2.480 \times 10^{6}$ \\
${ }^{136} \mathrm{Xe}$ & 42.05 & -47.99 & 54.77 & -30.60 & 9509 & 88.69 & $2.581 \times 10^{6}$ \\
\hline${ }^{\mathrm{Nucleus}}$ & $D_{1}$ & $D_{0}$ & $D_{1+}$ & $D_{2}$ & $D_{3}$ & $D_{4}$ & $D_{5}$ \\
\hline${ }^{76} \mathrm{Ge}$ & 2.873 & -5.041 & 8.846 & 0.7080 & -12.21 & -2.634 & $-2.309 \times 10^{5}$ \\
${ }^{82} \mathrm{Se}$ & 52.13 & -21.50 & 8.867 & -11.42 & -4.815 & -186.5 & $-2.154 \times 10^{5}$ \\
${ }^{100} \mathrm{Mo}$ & 0.1593 & -5.062 & 160.9 & -0.5702 & -26.72 & -27.92 & $-5.389 \times 10^{6}$ \\
${ }^{130} \mathrm{Te}$ & 26.17 & -37.97 & 55.09 & -4.971 & -124.1 & -63.12 & $-1.951 \times 10^{6}$ \\
${ }^{136} \mathrm{Xe}$ & 35.50 & -40.52 & 46.25 & -4.889 & -165.0 & -70.31 & $-2.014 \times 10^{6}$ \\
\hline
\end{tabular}

Table 5. Ratios of the half-lives, $\mathcal{R}$ and $\mathcal{R}_{\alpha}^{\beta}$, for various nuclei according to calculations on the basis of the QRPA model with (without) pn pairing [25]

\begin{tabular}{l|l|r|c|l}
\hline Nucleus & $\mathcal{R}=\mathcal{R}_{V-A}^{V-A}$ & \multicolumn{1}{c|}{$\mathcal{R}_{V+A}^{V-A}$} & $\mathcal{R}_{V-A}^{V+A}$ & \multicolumn{1}{c}{$\mathcal{R}_{V+A}^{V+A}$} \\
\hline${ }^{82} \mathrm{Se}$ & $0.4(0.2)$ & $0.4(2.8)$ & $2.1(3.1)$ & $0.3(0.03)$ \\
${ }^{100} \mathrm{Mo}$ & $1.1(195.2)$ & $52.9(0.6)$ & $1.1(0.5)$ & $1.1(0.8)$ \\
${ }^{130} \mathrm{Te}$ & $0.2(0.1)$ & $0.2(0.2)$ & $0.2(0.1)$ & $0.2(0.04)$ \\
${ }^{136} \mathrm{Xe}$ & $0.5(0.2)$ & $0.4(0.4)$ & $0.4(0.2)$ & $0.4(0.1)$ \\
\hline
\end{tabular}

$$
K=D_{1} / C_{1}=B_{01} / A_{01} .
$$

Here, $C_{1}=A_{01}\left(1-\chi_{\mathrm{F}}\right)^{2}$, where $\chi_{\mathrm{F}}=\left(g_{V} / g_{A}\right)^{2} \times$ $M_{\mathrm{F}} / M_{\mathrm{GT}}$, with $M_{\mathrm{F}}$ being the Fermi nuclear matrix element and $g_{V}$ and $g_{A}$ being, respectively, the vector and axial-vector nucleon form factors, and $A_{01}$ and $B_{01}$ are kinematical factors (integrals over the electron phase space) [22]. Taking into account the dipole momentum-transfer dependence of the form factors $g_{V}$ and $g_{A}$ at various values of the cutoff parameter $\left(\Lambda_{A}>\Lambda_{V}\right)$, we obtain the tensor contribution $M_{T}$ to the total nuclear matrix elements. As a result, $M^{0 \nu}=M_{\mathrm{GT}}\left(1-\chi_{\mathrm{F}}\right)$ is replaced by a more reliable form, $M^{\prime 0 \nu}=M^{0 \nu}+M_{T}$ (according to [27, 28]).

For the chosen set of nuclei and the mass interval of interest from the experimental point of view, the half-life dependences of the square of the effective Majorana mass [see Eq. (6)] in Fig. 2 for the nuclear matrix element $M^{0 \nu}\left(M^{\prime 0 \nu}\right)$ calculated on the basis of the QRPA model without and with allowance for $p n$ pairing [25] (RQRPA and QRPA models in [28]) are represented by, respectively, the solid and long-dashed curves ( short-dashed and dotted curves). The two vertical straight lines in Fig. 2 indicate the planned sensitivity limits at the first and subsequent stages of future experiments aimed at searches for $0 \nu 2 \beta$ decay:

$$
\begin{aligned}
& T_{1 / 2}\left(\text { GERDA, Majorana, }{ }^{76} \mathrm{Ge}\right) \\
& =2 \times 10^{26}-4 \times 10^{27} \mathrm{yr}[29,30],
\end{aligned}
$$

$T_{1 / 2}\left(\right.$ SuperNEMO,$\left.{ }^{82} \mathrm{Se}\right)=(1-2) \times 10^{26} \mathrm{yr}[31,32]$, $T_{1 / 2}\left(\mathrm{MOON},{ }^{100} \mathrm{Mo}\right)=2.1 \times 10^{26}-1 \times 10^{27} \mathrm{yr}[33]$, $T_{1 / 2}\left(\right.$ COURE, $\left.{ }^{130} \mathrm{Te}\right)=(2.1-6.5) \times 10^{26} \mathrm{yr}[31,34]$, $T_{1 / 2}\left(\mathrm{EXO},{ }^{136} \mathrm{Xe}\right)=6.4 \times 10^{25}-2 \times 10^{27} \mathrm{yr}[31,35]$.

From Fig. 2, one can see that, for the chosen set of decaying nuclei and at a fixed value of the halflife $T_{1 / 2}$, the calculated effective Majorana mass $|\langle m\rangle|$ depends substantially on the choice of nuclear model. In particular, a limit on $|\langle m\rangle|^{2}$ for the ${ }^{76} \mathrm{Ge}$ nucleus is approximately five times more stringent in the case of the QRPA model without $p n$ pairing than in the case of the QRPA model with allowance for $p n$ pairing. On the other hand, Fig. 2 demonstrates the possibility of discriminating between the predictions of different nuclear models by using the results for several decaying nuclei. For all four of the nuclear models considered here, the ${ }^{130}$ Te nucleus shows the highest 

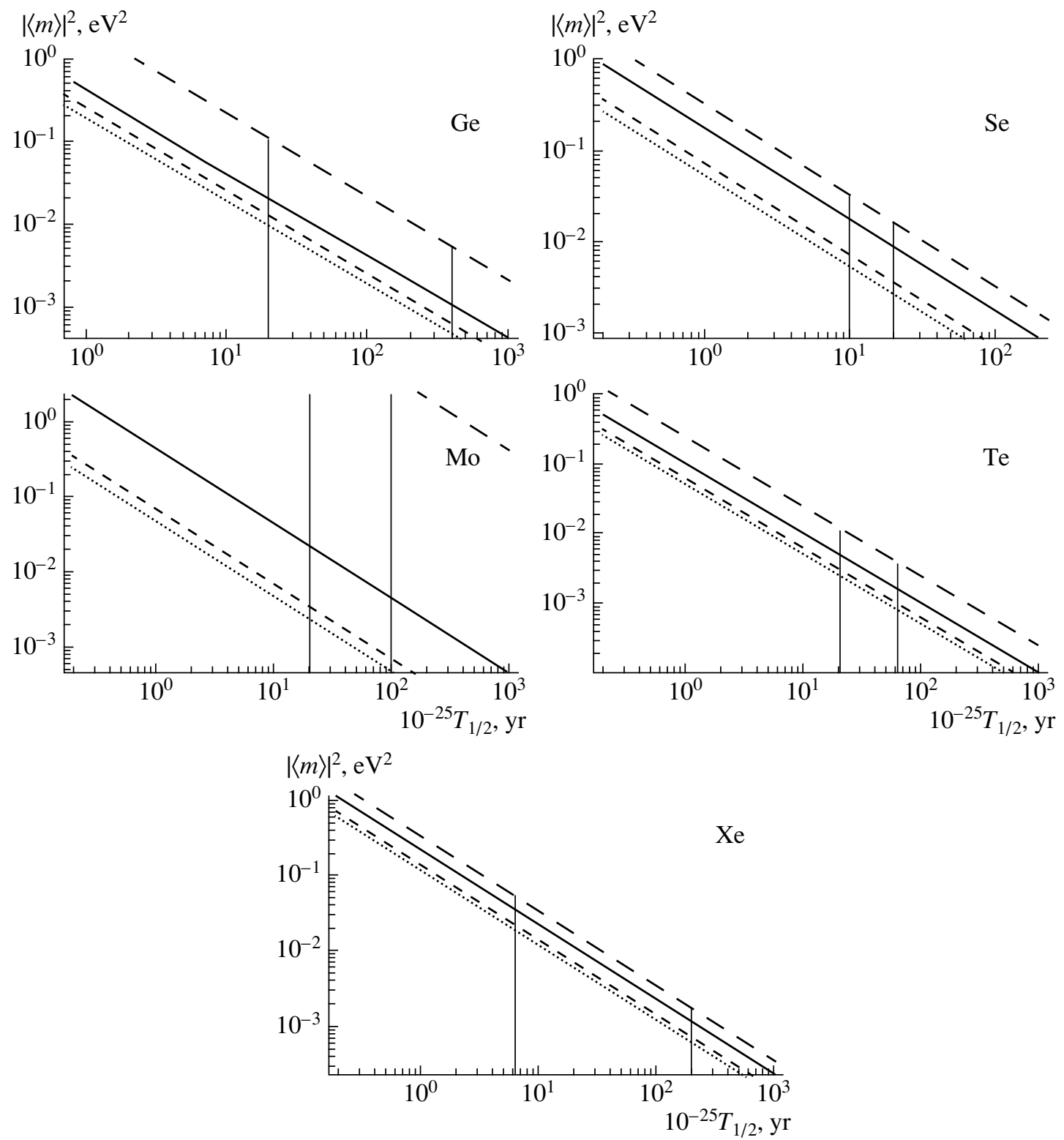

Fig. 2. Square of the effective Majorana mass, $|\langle m\rangle|^{2}$, as a function of the half-life $T_{1 / 2}$ for various nuclear models (see main body of the text).

sensitivity to the effective Majorana mass $|\langle m\rangle|$-it permits testing the smallest values of this mass (at a fixed value of $T_{1 / 2}$ ).

In order to compare the half-lives and angularcorrelation coefficients for different nuclei, we consider the ratios

$$
\mathcal{R}\left({ }^{A} X\right)=\frac{T_{1 / 2}\left({ }^{A} X\right)}{T_{1 / 2}\left({ }^{76} \mathrm{Ge}\right)}, \quad \mathcal{K}\left({ }^{A} X\right)=\frac{K\left({ }^{A} X\right)}{K\left({ }^{76} \mathrm{Ge}\right)},
$$

where, as a reference nucleus, we use the isotope ${ }^{76} \mathrm{Ge}$, which has received the most detailed study. Concurrently, we notice that relative quantities usually are less sensitive to the theoretical uncertainty in the nuclear matrix elements than their absolute counterparts (see [36] and references therein). The numerical values of the ratios in (7) for the standard decay mechanism $\left(\epsilon_{\alpha}^{\beta}=0,|\langle m\rangle| \neq 0\right)$ are presented in the second columns of Tables 5 and 6 .

(B) $\boldsymbol{\epsilon}_{\boldsymbol{\alpha}}^{\boldsymbol{\beta}} \neq \mathbf{0},|\langle m\rangle|=\mathbf{0}$. By analogy with (7), we define the ratios

$$
\begin{gathered}
\mathcal{R}_{\alpha}^{\beta}\left({ }^{A} X\right)=\frac{T_{1 / 2}\left(\epsilon_{\alpha}^{\beta},{ }^{A} X\right)}{T_{1 / 2}\left(\epsilon_{\alpha}^{\beta},{ }^{76} \mathrm{Ge}\right)}, \\
\mathcal{K}_{\alpha}^{\beta}\left({ }^{A} X\right)=\frac{K\left(\epsilon_{\alpha}^{\beta},{ }^{A} X\right)}{K\left(\epsilon_{\alpha}^{\beta},{ }^{76} \mathrm{Ge}\right)},
\end{gathered}
$$


Table 6. Ratios of the angular correlation coefficients $\mathcal{K}$ and $\mathcal{K}_{\alpha}^{\beta}$ for various nuclei in the QRPA model without (with) allowance for $p n$ pairing [25]

\begin{tabular}{l|c|c|c|c|c}
\hline Nucleus & $\mathcal{K}=\mathcal{K}_{V \pm A}^{V-A}$ & $\mathcal{K}_{V-A}^{V+A}$ & $\left.\left.\frac{B_{09}}{A_{09}}\right|_{A X} \frac{A_{09}}{B_{09}}\right|_{76 \mathrm{Ge}}$ & $\mathcal{K}_{V+A}^{V+A}$ & $\left.\left.\frac{B_{02}}{A_{02}}\right|_{A_{X}} \frac{A_{02}}{B_{02}}\right|_{76 \mathrm{Ge}}$ \\
\hline${ }^{82} \mathrm{Se}$ & 1.08 & $1.12(1.12)$ & 1.13 & $1.13(0.95)$ & 1.06 \\
${ }^{100} \mathrm{Mo}$ & 1.08 & $1.15(1.15)$ & 1.13 & $1.14(0.85)$ & 1.05 \\
${ }^{130} \mathrm{Te}$ & 1.04 & $1.07(1.07)$ & 1.07 & $1.01(0.90)$ & 1.01 \\
${ }^{136} \mathrm{Xe}$ & 1.04 & $1.07(1.07)$ & 1.06 & $0.98(0.91)$ & 1.01 \\
\hline
\end{tabular}

Table 7. Numerical values of $\lambda_{i}$ [in units of $10^{12} \mathrm{yr}$ ] for $\epsilon_{V+A}^{V+A} \neq 0$

\begin{tabular}{l|c|c|c|c}
\hline Nucleus & $\lambda_{1}$ & $\lambda_{2}$ & $\lambda_{3}$ & \multicolumn{1}{c}{$\lambda_{4}$} \\
\hline${ }^{76} \mathrm{Ge}$ & 7.942 & -10.10 & -5.140 & -6.310 \\
${ }^{82} \mathrm{Se}$ & 3.429 & -3.846 & -1.246 & -1.413 \\
${ }^{100} \mathrm{Mo}$ & 8.796 & -9.840 & -5.359 & -6.072 \\
${ }^{130} \mathrm{Te}$ & 1.917 & -2.403 & -0.8231 & -0.9698 \\
${ }^{136} \mathrm{Xe}$ & 4.112 & -5.315 & -1.824 & -2.161 \\
\hline
\end{tabular}

which characterize the contributions of non-SM decay mechanisms individually (only one of the coefficients $\epsilon_{\alpha}^{\beta}$ is nonzero). These ratios do not depend on $\epsilon_{\alpha}^{\beta}$ only for $|\langle m\rangle|=0$, but they depend on $\alpha$ and $\beta$ through various sets of coefficients $C_{i}$ and $D_{i}$ appearing in the expressions for these ratios (see Table 2). The values of $\mathcal{R}_{\alpha}^{\beta}$ and $\mathcal{K}_{\alpha}^{\beta}$ for the chosen nuclei are presented in Tables 5 and 6 . The ratios of the half-lives are markedly different, with the exception of the ratios $\mathcal{R}_{V-A}^{V-A}$ coinciding with the ratio $\mathcal{R}$ for the standard decay mechanism. However, these variations are largely due to theoretical uncertainties in the nuclear matrix elements. This is illustrated in Table 5 by comparing the results of the calculations within the QRPA model with and without allowance for $p n$ pairing. In particular, the ratio $\mathcal{R}_{V+A}^{V-A}$ $\left(\mathcal{R}=\mathcal{R}_{V-A}^{V-A}\right)$ for the ${ }^{100}$ Mo nucleus is two orders of magnitude larger than the analogous ratios for the other nuclei in the QRPA model without (with) allowance for $p n$ pairing. This is because the ratio $\mathcal{R}_{V+A}^{V-A}\left({ }^{A} X\right)\left(\mathcal{R}=\mathcal{R}_{V-A}^{V-A}\left({ }^{A} X\right)\right)$ is proportional to the nuclear-matrix-element combination $\left|M_{\mathrm{GT}}\right|^{-2} \mid \chi_{\mathrm{F}}+$ $\left.1\right|^{-2}\left(\left|M_{\mathrm{GT}}\right|^{-2}\left|\chi_{\mathrm{F}}-1\right|^{-2}\right)$, with $\left|M_{\mathrm{GT}}\right|$ and $\left|\chi_{\mathrm{F}}+1\right|$ $\left(\left|M_{\mathrm{GT}}\right|\right.$ and $\left.\left|\chi_{\mathrm{F}}-1\right|\right)$ being smaller for the ${ }^{100} \mathrm{Mo}$ nucleus than for the other nuclei by factors of about 3 (2) and 5 (10), respectively: only for the ${ }^{100}$ Mo nucleus is the value of $\chi_{\mathrm{F}}$ accidentally close to $-1(+1)$. We
Table 8. Numerical values of $\lambda_{1,2}$ [in units of $10^{12} \mathrm{yr}$ ] and $\lambda_{3,4}$ [in units of $10^{8} \mathrm{yr}$ ] for $\epsilon_{V-A}^{V+A} \neq 0$

\begin{tabular}{l|c|c|c|c}
\hline Nucleus & $\lambda_{1}$ & $\lambda_{2}$ & $\lambda_{3}$ & $\lambda_{4}$ \\
\hline${ }^{76} \mathrm{Ge}$ & 7.657 & -10.45 & -1.947 & -2.390 \\
${ }^{82} \mathrm{Se}$ & 3.286 & -4.008 & -4.030 & -4.573 \\
${ }^{100} \mathrm{Mo}$ & 8.518 & -10.16 & -2.109 & -2.390 \\
${ }^{130} \mathrm{Te}$ & 1.903 & -2.419 & -0.3864 & -0.4553 \\
${ }^{136} \mathrm{Xe}$ & 4.130 & -5.293 & -0.7885 & -0.9338 \\
\hline
\end{tabular}

note that, for $\mathcal{R}_{V \mp A}^{V+A}$, there is no similar accidental enhancement because of a more intricate structure of the corresponding expressions \{see expressions (6) and (7) and Table 2 above; see also Eq. (28) in [22]\}.

On the other hand, the ratios of the angularcorrelation coefficients $\mathcal{K}$ and $\mathcal{K}_{V \pm A}^{V-A}$ do not depend on the nuclear matrix elements and assume the form

$$
\mathcal{K}=\mathcal{K}_{V-A}^{V-A}=\mathcal{K}_{V+A}^{V-A}=\left.\left.\frac{B_{01}}{A_{01}}\right|_{A_{X}} \frac{A_{01}}{B_{01}}\right|_{7{ }^{6} \mathrm{Ge}} .
$$

Also, the coefficients $\mathcal{K}_{V \pm A}^{V+A}$ are virtually independent of the uncertainties in the nuclear matrix elements, because the following approximate relations hold:

$$
\begin{aligned}
& \left.\left.\mathcal{K}_{V-A}^{V+A} \simeq \frac{B_{09}}{A_{09}}\right|_{A_{X}} \frac{A_{09}}{B_{09}}\right|_{76 \mathrm{Ge}}, \\
& \left.\left.\mathcal{K}_{V+A}^{V+A} \simeq \frac{B_{02}}{A_{02}}\right|_{A_{X}} \frac{A_{02}}{B_{02}}\right|_{{ }^{6} \mathrm{Ge}} .
\end{aligned}
$$

We recall that, here, $A_{i}$ and $B_{i}$ are purely kinematical quantities (phase-space integrals). The values of the right-hand sides of Eqs. (10) are given in the fourth and sixth columns of Table 6 for the sake of comparison. We note, however, that these ratios of the angular-correlation coefficients do not provide a possibility for discriminating between the predictions of the different theories since, within the assumed experimental error of about $20 \%$, their values are compatible with unity. 

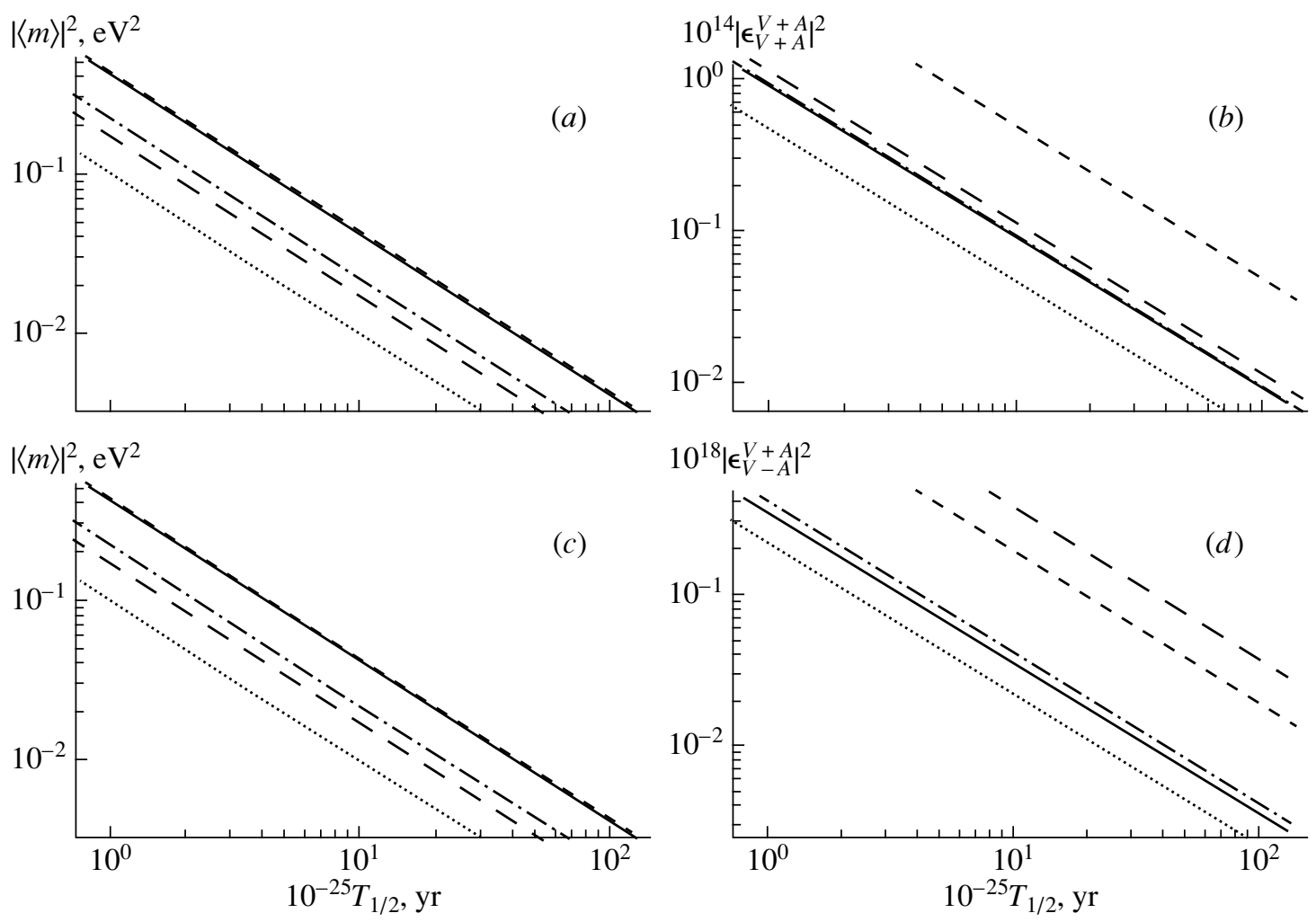

Fig. 3. Correlations $(a, c)$ between $|\langle m\rangle|$ and $T_{1 / 2}$ and $(b, d)$ between $\left|\epsilon_{\alpha}^{\beta}\right|$ and $T_{1 / 2}$ for a fixed value of $K=0.8$ and a nonzero value of the parameter $(a, b) \epsilon_{V+A}^{V+A}$ or $(c, d) \epsilon_{V-A}^{V+A}$. The solid, long-dashed, short-dashed, dotted, and dash-dotted curves represent the results for the decays of the ${ }^{76} \mathrm{Ge},{ }^{82} \mathrm{Se},{ }^{100} \mathrm{Mo},{ }^{130} \mathrm{Te}$, and ${ }^{136} \mathrm{Xe}$ nuclei, respectively.

The ${ }^{100}$ Mo nucleus shows the highest sensitivity to non-SM decay mechanisms in terms of the properties considered here; this is not so only in the case of the ratio $\mathcal{R}_{V-A}^{V+A}$, for which the ${ }^{82}$ Se nucleus is the most sensitive.

According to half-life measurements, the ${ }^{100} \mathrm{Mo}-$ ${ }^{130} \mathrm{Te}$ and ${ }^{82} \mathrm{Se}-{ }^{130} \mathrm{Te}$ pairs of nuclei are the most sensitive to the effects of the non-SM parameters $\epsilon_{V+A}^{V \pm A}$ and $\epsilon_{V-A}^{V+A}$, respectively.

Measurements of angular-correlation coefficients show that the ${ }^{76} \mathrm{Ge}-{ }^{100}$ Mo pair of nuclei is the most sensitive to the effects of $\epsilon_{V \pm A}^{V+A}$.

(C) $\epsilon_{\boldsymbol{\alpha}}^{\boldsymbol{\beta}} \neq 0,|\langle m\rangle| \neq 0, c_{i}=0$. With the aid of Eqs. (4) and (6) and the data in Table 2, the reduced Majorana mass $\mu=\langle m\rangle / m_{e}$ and the parameter $\epsilon_{\alpha}^{\beta}$ of the non-SM Majorana mass $\mu_{\alpha}^{\beta}$ (according to the choice of indices $\alpha$ and $\beta$ in Table 2) can be expressed in terms of the half-life $T_{1 / 2}$ and the angular-correlation coefficient $K$ (which are measurable quantities) as

$$
\begin{gathered}
|\mu|^{2}=\left(\lambda_{1}-\lambda_{2} K\right) / T_{1 / 2}, \\
\left|\epsilon_{\alpha}^{\beta}\right|^{2}\left(\operatorname{or} 4\left|\mu_{\alpha}^{\beta}\right|^{2}\right)=\left(-\lambda_{3}+\lambda_{4} K\right) / T_{1 / 2},
\end{gathered}
$$

where the coefficients are

$$
\begin{aligned}
& \lambda_{1}=D_{i} / \Delta_{i}, \quad \lambda_{2}=C_{i} / \Delta_{i}, \\
& \lambda_{3}=D_{1} / \Delta_{i}, \quad \lambda_{4}=C_{1} / \Delta_{i}
\end{aligned}
$$

with $\Delta_{i}=\left|M_{\mathrm{GT}}\right|^{2}\left(C_{1} D_{i}-D_{1} C_{i}\right)$. In particular, we have $i=4(5)$ for $\epsilon_{V+A}^{V+A}\left(\epsilon_{V-A}^{V+A}\right)$.

The values of $\lambda_{i}$ for the nuclei considered here are given in Table 7 (8) for a nonzero value of the parameter $\epsilon_{V+A}^{V+A}\left(\epsilon_{V-A}^{V+A}\right)$.

Figures $3,4,5,6$, and 7 show correlations between $|\langle m\rangle|$ and $T_{1 / 2}$ and between $\left|\epsilon_{\alpha}^{\beta}\right|$ and $T_{1 / 2}$ for the chosen nuclei in the QRPA model without allowance for $p n$ pairing [25] at a nonzero value of the parameter $(a$, b) $\epsilon_{V+A}^{V+A}$ or $(c, d) \epsilon_{V-A}^{V+A}$ for the angular-correlation coefficient fixed at $K=0.8,0.4,0,-0.4$, and -0.8 , respectively. Figure 8 shows correlations between $|\langle m\rangle|$ and $K$ and between $\left|\epsilon_{\alpha}^{\beta}\right|$ and $K$ for the same nuclei and at a fixed half-life value of $T_{1 / 2}=10^{25} \mathrm{yr}$ for nonzero $\epsilon_{V+A}^{V+A}$ or $\epsilon_{V-A}^{V+A}$. The case of $K=0.8$ (Fig. 3) approximately reproduces the standard decay mechanism (SM + Majorana neutrino) considered in Section (A)-see Fig. 2. From Figs. 3-7, one can see that, smaller values of $K$ correspond to larger values 

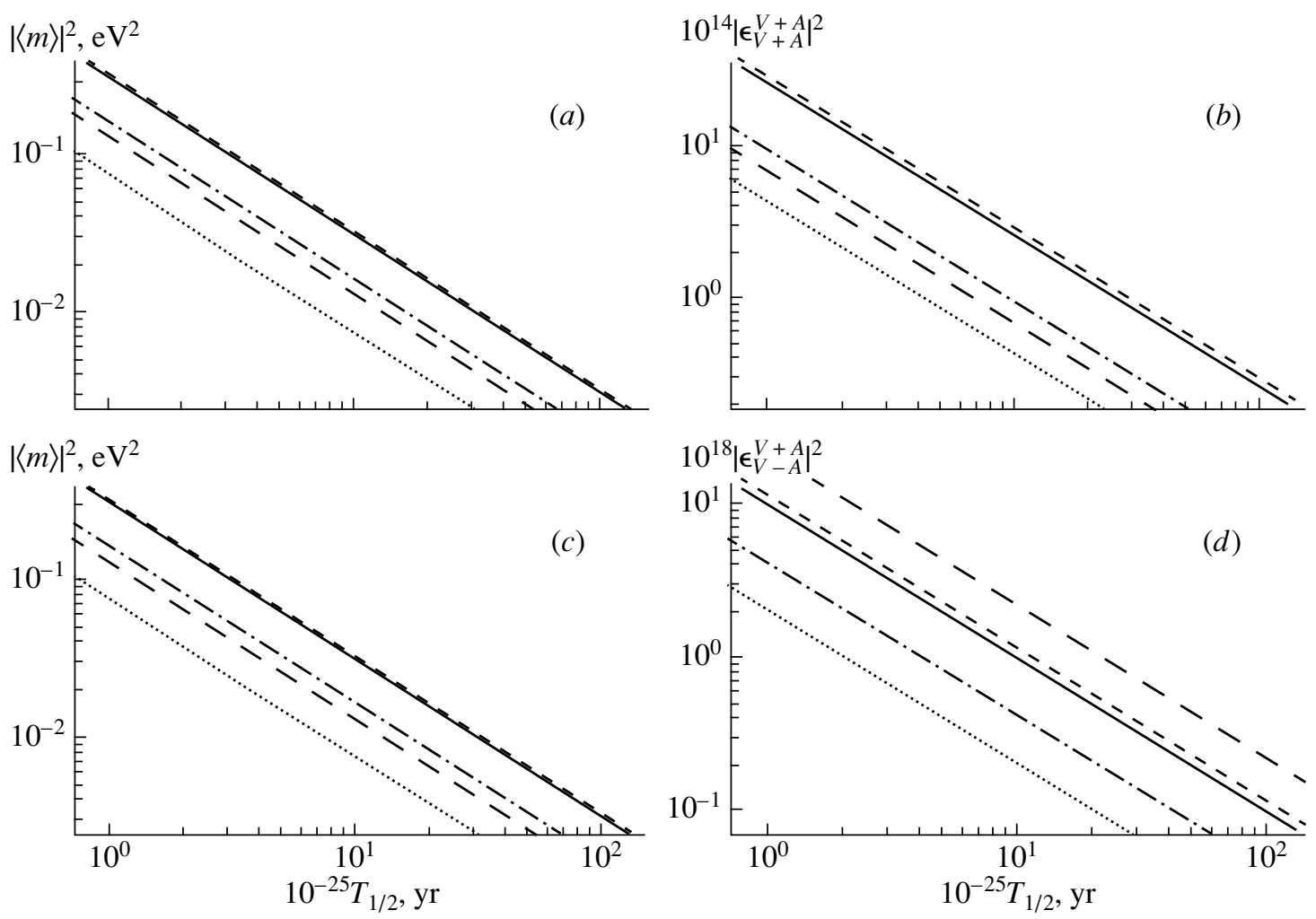

Fig. 4. As in Fig. 3, but for $K=0.4$.
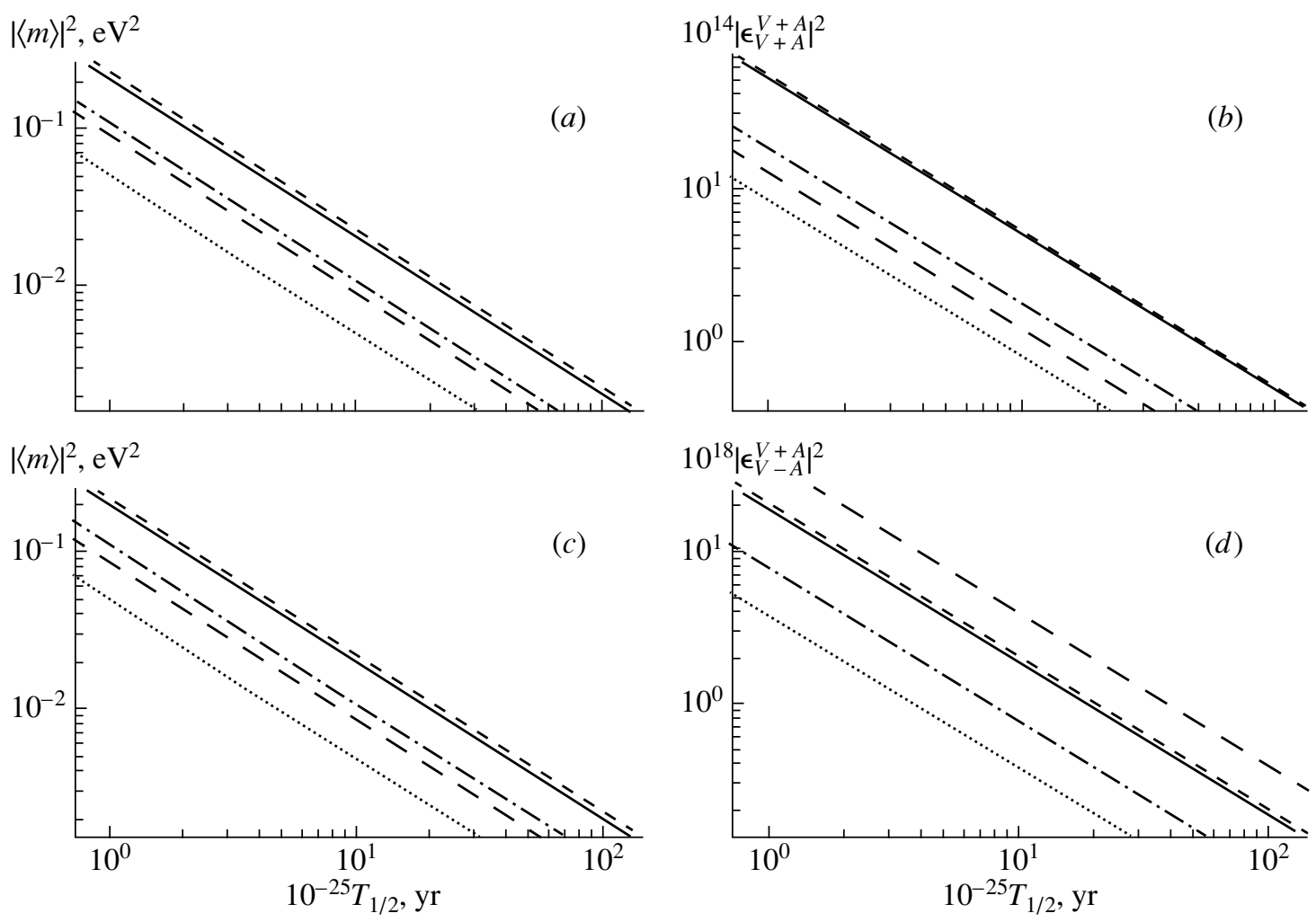

Fig. 5. As in Fig. 3, but for $K=0$. 

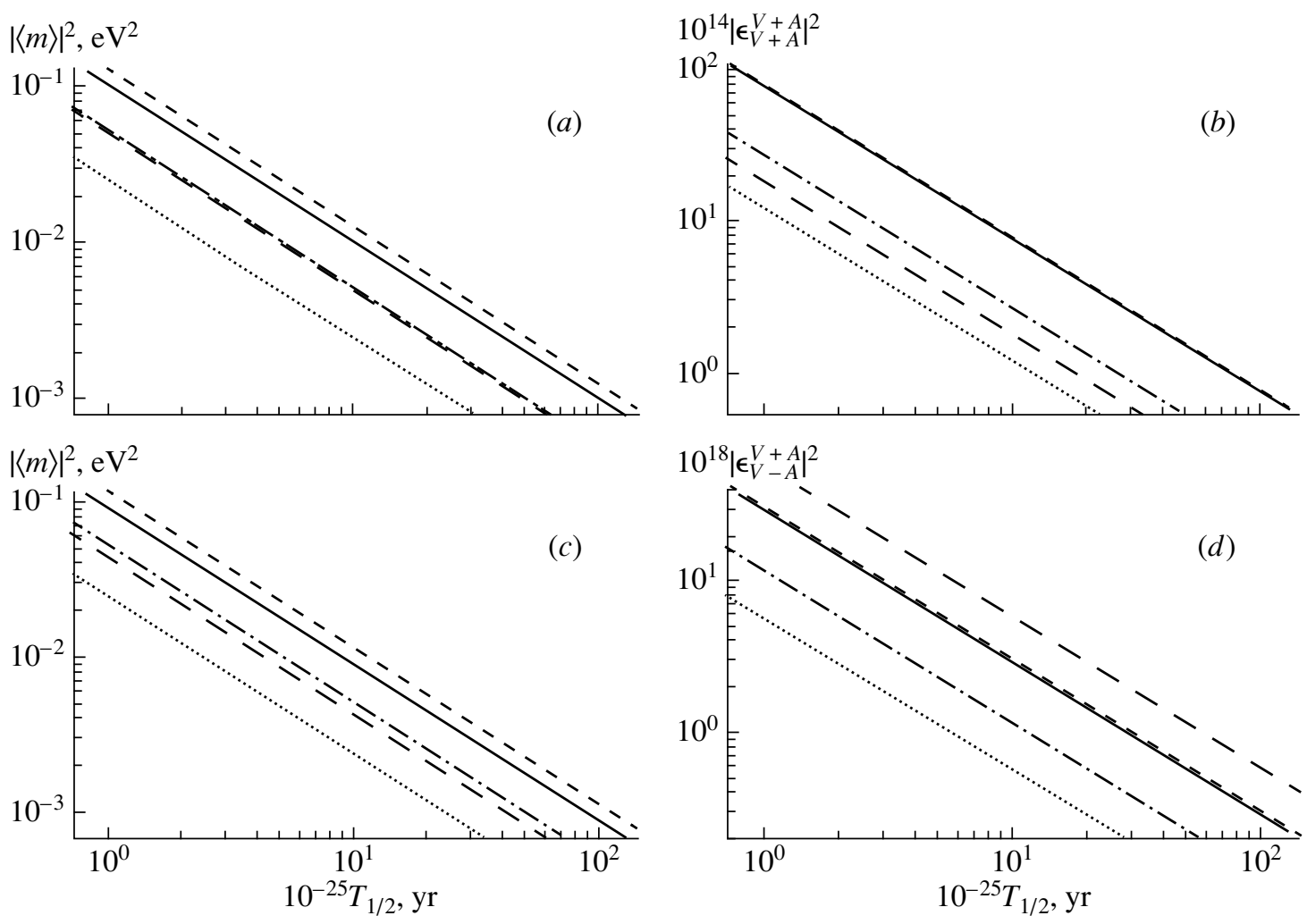

Fig. 6. As in Fig. 3, but for $K=-0.4$.
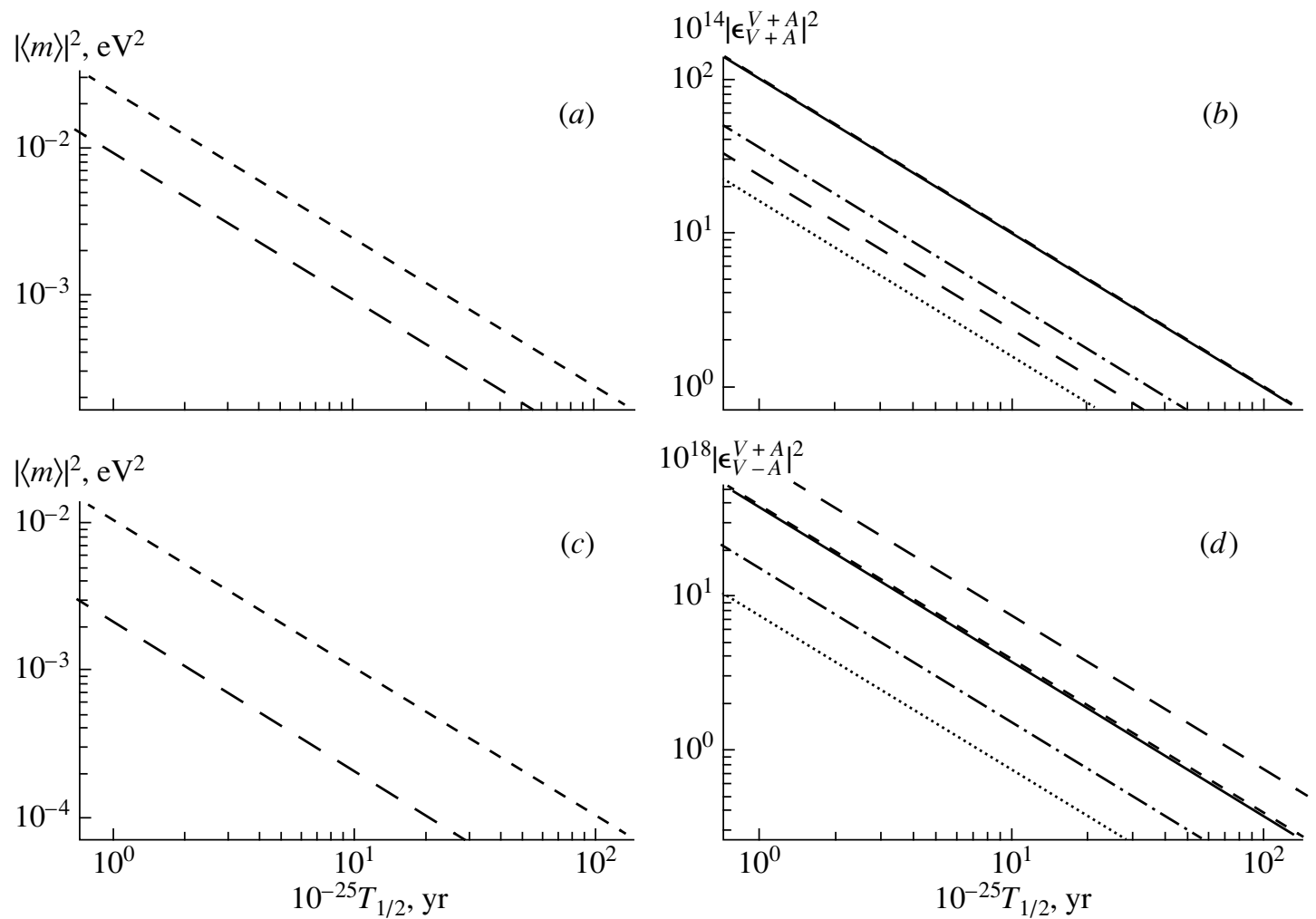

Fig. 7. As in Fig. 3, but for $K=-0.8$. 

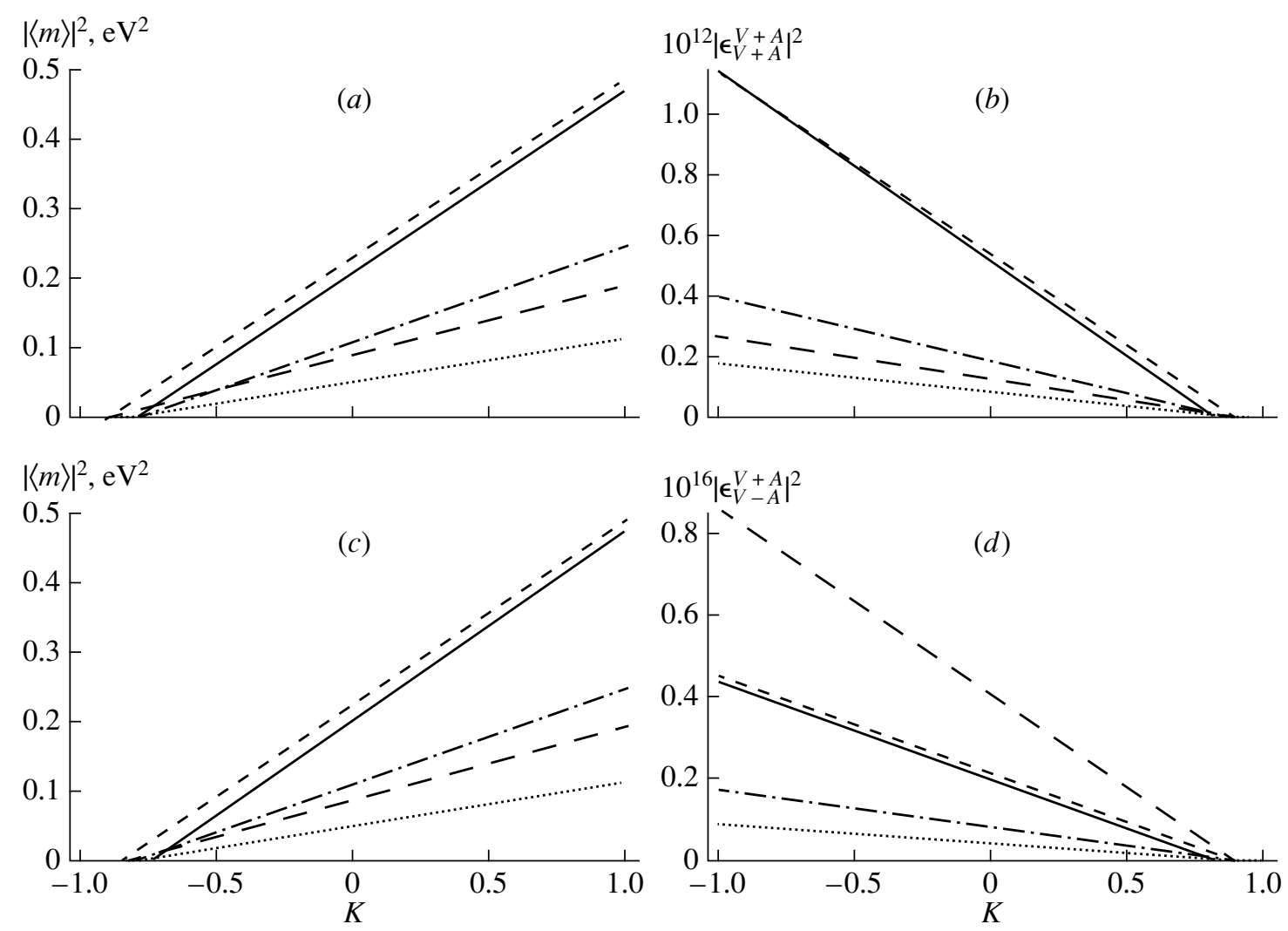

Fig. 8. Correlations $(a, c)$ between $|\langle m\rangle|$ and $K$ and $(b, d)$ between $\left|\epsilon_{\alpha}^{\beta}\right|$ and $K$ for a fixed value of $T_{1 / 2}=10^{25}$ yr and a nonzero value of the parameter $(a, b) \epsilon_{V+A}^{V+A}$ or $(c, d) \epsilon_{V-A}^{V+A}$. The notation for the curves is identical to that in Fig. 3.

of $\epsilon_{V \pm A}^{V+A}$, the ${ }^{76} \mathrm{Ge},{ }^{82} \mathrm{Se}$, and ${ }^{100}$ Mo nuclei being the most sensitive to angular correlations. Figures $3 a$ and $3 c$ are almost identical (the same is true for Figs. 46 and 8$)$, because the values of $\lambda_{1,2}$ at $\epsilon_{V+A}^{V+A} \neq 0$ are very close to those at $\epsilon_{V-A}^{V+A} \neq 0$ for a fixed nucleus; the difference is significant only at small values of $|\langle m\rangle|$ (Fig. 7). In Figs. $7 a$ and $7 c$, there are curves only for the ${ }^{82} \mathrm{Se}$ and ${ }^{100} \mathrm{Mo}$ nuclei since, for the remaining nuclei, ${ }^{76} \mathrm{Ge},{ }^{130} \mathrm{Te}$, and ${ }^{136} \mathrm{Xe}$, the values of $|\langle m\rangle|$ are overly small. We note that, in Figs. 6 and 7 ( $b$ and $d$ ), the curves corresponding to the ${ }^{76} \mathrm{Ge}$ and ${ }^{100} \mathrm{Mo}$ nuclei coincide with each other.

Figure 9 illustrates correlations between $(a, c, e)$ the mass $M_{W_{R}}$ of the right-handed $W_{R}$ boson, $(b$, $d, f)$ the mixing angle $\zeta,(a-d)$ the half-life $T_{1 / 2}$, and $(e, f)$ the angular-correlation coefficient $K$ within the $S U(2)_{L} \times S U(2)_{R} \times U(1)$ left-right symmetry model $[22,37]$ for the case of equal form factors for left- and right-handed hadron currents $\left(g_{V}=\right.$ $\left.g_{V}^{\prime}\right)$ and the mixing-parameter value of $\epsilon=\left|U_{e i} V_{e i}\right|=$ $10^{-6}[22]$.

From Fig. 9, one can see that, the larger $T_{1 / 2}$ at fixed $K$ or the closer $K$ to unity at fixed $T_{1 / 2}$, the more stringent the lower limit on $M_{W_{R}}$ (upper limit on $\zeta$ ), the maximum being reached for the ${ }^{130} \mathrm{Te}$ nucleus. However, a significant effect of the $W_{R}$ boson on $0 \nu 2 \beta$ decay for $T_{1 / 2}<10^{26} \mathrm{yr}$ is impossible because of a stringent limit on its mass from an electroweak fit [24]: $M_{W_{R}}>715 \mathrm{GeV}$. We note that, in Figs. $9 c$ and $9 d$, the curves corresponding to the ${ }^{76} \mathrm{Ge}$ and ${ }^{100}$ Mo nuclei merge together.

(D) $\epsilon_{\boldsymbol{\alpha}}^{\boldsymbol{\beta}} \neq 0,|\langle m\rangle| \neq 0, \boldsymbol{c}_{\boldsymbol{i}} \neq \mathbf{0}$. Using relations (4) and (6) and the data from Table 2 , we obtain

$$
\begin{aligned}
& \mathcal{A}=C_{1}|\mu|^{2}+4 C_{i}|\mu|\left|\mu_{\alpha}^{\beta}\right| c_{0 k}+4 C_{j}\left|\mu_{\alpha}^{\beta}\right|^{2}, \\
& \mathcal{A} K=D_{1}|\mu|^{2}+4 D_{i^{\prime}}|\mu|\left|\mu_{\alpha}^{\beta}\right| c_{0 k}+4 D_{j^{\prime}}\left|\mu_{\alpha}^{\beta}\right|^{2}
\end{aligned}
$$

for $\beta=S-P, V-A, T_{L}$ (with the exception of $\alpha=$ $T_{R}$ and $\beta=T_{L}$ ) and

$$
\begin{aligned}
& \mathcal{A}=C_{1}|\mu|^{2}+C_{i}|\mu|\left|\epsilon_{\alpha}^{\beta}\right| c_{k}+C_{j}\left|\epsilon_{\alpha}^{\beta}\right|^{2}, \\
& \mathcal{A} K=D_{1}|\mu|^{2}+D_{i^{\prime}}|\mu|\left|\epsilon_{\alpha}^{\beta}\right| c_{k}+D_{j^{\prime}}\left|\epsilon_{\alpha}^{\beta}\right|^{2}
\end{aligned}
$$

for $\beta=S+P, V+A, T_{R}$ (with the exception of $\alpha=$ $T_{L}$ and $\left.\beta=T_{R}\right)$. Here, we have $\mathcal{A}=\left(\left|M_{\mathrm{GT}}\right|^{2} T_{1 / 2}\right)^{-1}$ [see Eq. (6)]. In the particular case of a nonzero value of the parameter $\epsilon_{V+A}^{V+A}\left(\epsilon_{V-A}^{V+A}\right)$, we have $i=i^{\prime}=2$, $j=j^{\prime}=4$, and $k=1\left(i=i^{\prime}=3, j=j^{\prime}=5, k=2\right)$. 

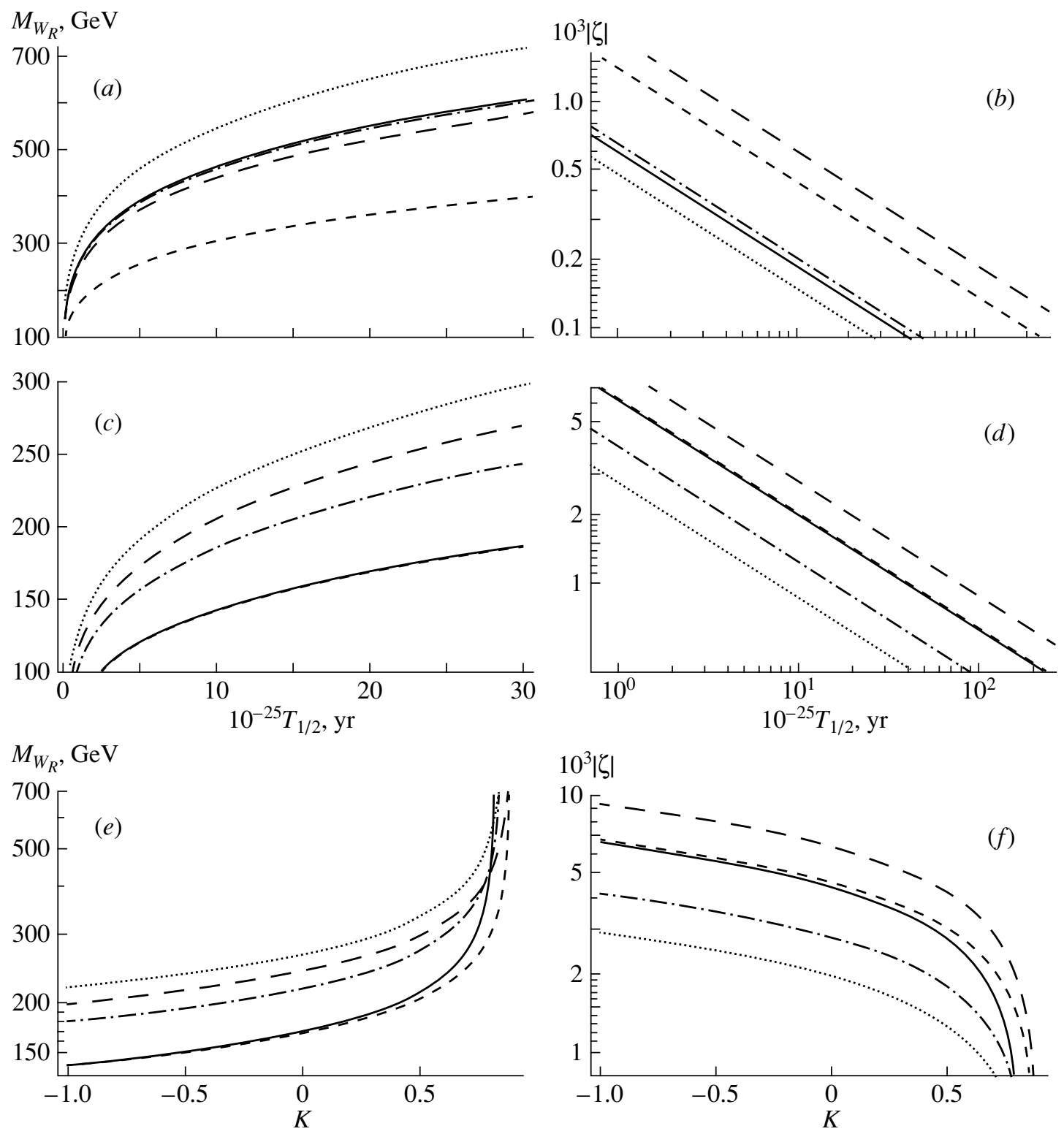

Fig. 9. Correlations between $(a, c, e) M_{W_{R}},(b, d, f) \zeta$ and $(a-d) T_{1 / 2},(e, f) K$ at $(a, b) K=0.8,(c, d) K=-0.8$, and $(e, f)$ $T_{1 / 2}=10^{26} \mathrm{yr}$. The notation for the curves is identical to that in Fig. 3.

The correlation between $\left|\epsilon_{V+A}^{V+A}\right|$ and $|\langle m\rangle|$ at the fixed values of $c_{1}=-1,0$, and 1 is shown in Fig. 10. In order to illustrate the fact that the results depend significantly on the theoretical uncertainty in the nuclear matrix elements [28, 38], we use, as before, two versions of the QRPA model: without and with allowance for $p n$ pairing [25]. One can readily see that a transition from one model version to the other leads predominantly to a parallel shift of the curves, which is maximal (minimal) for the ${ }^{100} \mathrm{Mo}\left({ }^{136} \mathrm{Xe}\right)$ nucleus.

It is obvious that, in the case of an experiment with a single isotope, the set of two Eqs. (13) or (14) gives no way to determine three quantities: $\left\{|\mu|, c_{0 i},\left|\mu_{\alpha}^{\beta}\right|\right\}$ or $\left\{|\mu|, c_{i}, \epsilon_{\alpha}^{\beta}\right\}$. For this, it is necessary to measure the half-lives of three isotopes or the half-lives of two isotopes and the angular-correlation coefficient $K$ for at least one of them. We consider in detail the second possibility, supplementing the set of Eqs. (13) or (14) for one nucleus whose angular-correlation coefficient $K$ was measured with the equation

$$
\tilde{\mathcal{A}}=\tilde{C}_{1}|\mu|^{2}+4 \tilde{C}_{i}|\mu|\left|\mu_{\alpha}^{\beta}\right| c_{0 k}+4 \tilde{C}_{j}\left|\mu_{\alpha}^{\beta}\right|^{2}
$$

or with the equation

$$
\tilde{\mathcal{A}}=\tilde{C}_{1}|\mu|^{2}+\tilde{C}_{i}|\mu|\left|\epsilon_{\alpha}^{\beta}\right| c_{k}+\tilde{C}_{j}\left|\epsilon_{\alpha}^{\beta}\right|^{2}
$$

for another nucleus whose coefficient $K$ is unknown. 


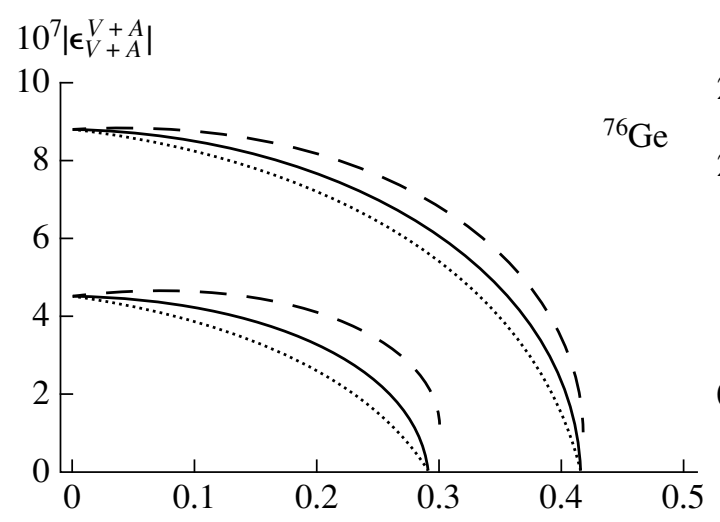

$$
10^{7}\left|\epsilon_{V+A}^{V+A}\right|
$$
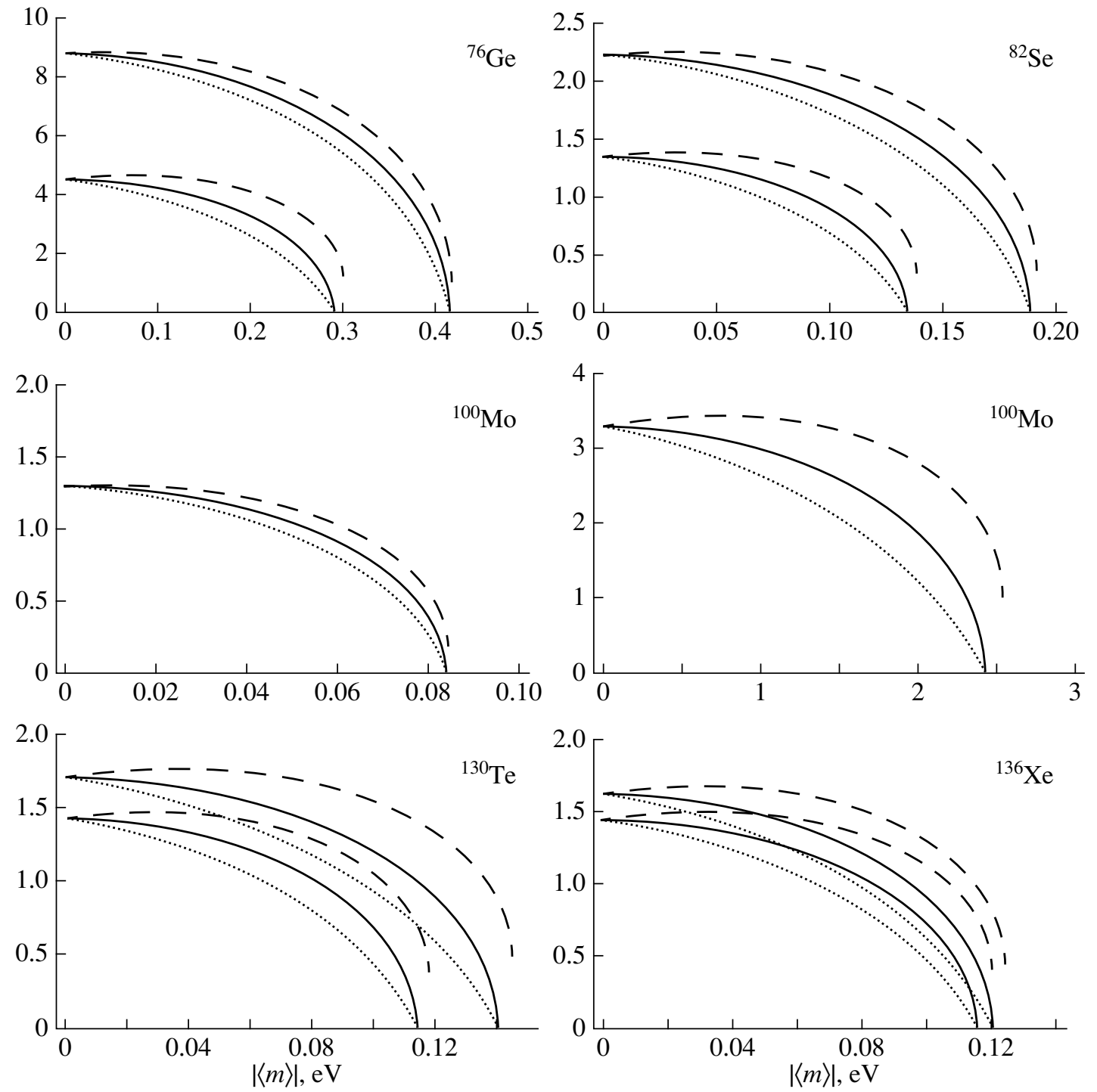

Fig. 10. Correlation between $\left|\epsilon_{V+A}^{V+A}\right|$ and $|\langle m\rangle|$ at $c_{1}=-1,0,1$ (dotted, solid, dashed curves, respectively) for various nuclei; three curves below on the left (above on the right) correspond to the QRPA model without (with) allowance for $p n$ pairing.

From (14) and (16), we obtain

$$
\begin{aligned}
|\mu| & =\sqrt{\frac{\Delta_{\mu}}{\Delta}}, \quad\left|\epsilon_{\alpha}^{\beta}\right|=\sqrt{\frac{\Delta_{\epsilon}}{\Delta}}, \\
c_{k} & =\frac{\mathcal{A} \Delta-C_{1} \Delta_{\mu}-C_{j} \Delta_{\epsilon}}{C_{i} \sqrt{\Delta_{\mu} \Delta_{\epsilon}}},
\end{aligned}
$$

where

$$
\begin{gathered}
\Delta=\lambda_{11} \lambda_{22}-\lambda_{12} \lambda_{21} \\
\Delta_{\mu}=\eta_{1} \lambda_{22}-\eta_{2} \lambda_{12}, \quad \Delta_{\epsilon}=\eta_{2} \lambda_{11}-\eta_{1} \lambda_{21}
\end{gathered}
$$

with the coefficients being

$$
\begin{gathered}
\lambda_{11}=C_{1}-C_{j} \tilde{C}_{1} / \tilde{C}_{j}, \\
\lambda_{12}=C_{i}-C_{j} \tilde{C}_{i} / \tilde{C}_{j}, \quad \eta_{1}=\mathcal{A}-\tilde{A} C_{j} / \tilde{C}_{j},
\end{gathered}
$$

$$
\begin{gathered}
\lambda_{21}=D_{1}-D_{j^{\prime}} \tilde{C}_{1} / \tilde{C}_{j}, \quad \lambda_{22}=D_{i^{\prime}}-D_{j^{\prime}} \tilde{C}_{i} / \tilde{C}_{j}, \\
\eta_{2}=\mathcal{A} K-\tilde{A} D_{j^{\prime}} / \tilde{C}_{j} .
\end{gathered}
$$

Similar expressions can be obtained from Eqs. (13) and (15).

It follows that, having measured the half-lives of two isotopes and the angular-correlation coefficient for one of them, one can calculate, on the basis of Eqs. (17)-(19), the effective Majorana mass $|\langle m\rangle| \equiv$ $|\mu| m_{e}$ and the parameters $\left|\epsilon_{\alpha}^{\beta}\right|$ and $c_{i}$ characterizing non-SM decay mechanisms.

\section{CONCLUSIONS}

On the basis of an effective Lagrangian that describes long-range mechanisms of $0 \nu 2 \beta$ decay via 
the exchange of a light Majorana neutrino and heavy non-SM particles, we have analyzed half-lives and electron angular-correlation coefficients for the decays of several nuclei that will be used in future experiments. A comparison of the results of measurements for the above properties of decay of several nuclei will make it possible to minimize the theoretical uncertainties in the relevant nuclear matrix elements and to extract information about a dominant decay mechanism. Measurement of the angular-correlation coefficient for electrons will permit refining this information $[21,22]$. It should be noted that the angular distributions of electrons in the two-neutrino beta decay of the ${ }^{100} \mathrm{Mo}$ and ${ }^{82}$ Se nuclei have already been measured in the NEMO3 experiment, which is currently under way. In the future, it is planned to perform similar measurements for $0 \nu 2 \beta$ decay upon accumulating sufficiently large statistics of respective events [39]. Other planned experiments that will be able to measure the angular distributions of electrons include SuperNEMO [40], MOON [33, 41], and EXO [42].

\section{ACKNOWLEDGMENTS}

We are grateful to the reviewer of this article for enlightening comments.

\section{REFERENCES}

1. P. Minkowski, Phys. Lett. B 67, 421 (1977); T. Yanagida, in Proc. of the Workshop on the Unified Theories and the Baryon Number in the Universe, Ed. by O. Sawada and A. Sugamoto (Tsukuba, Japan, 1979), p. 95; M. Gell-Mann, P. Ramond, and R. Slansky, in Supergravity, Ed. by D. Z. Freedman and P. van Nieuwenhuizen (North-Holland, Amsterdam, 1979), p. 315; S. L. Glashow, in Proc. of the 1979 Cargese Summer Institute on Quarks and Leptons, Ed. by M. Levy et al. (New York, 1980), p. 687; R. N. Mohapatra and G. Senjanovic, Phys. Rev. Lett. 44, 912 (1980); R. N. Mohapatra et al., Rep. Prog. Phys. 70, 1757 (2007).

2. A. Zee, Phys. Lett. B 93, 389 (1980); Phys. Lett. B 95, 461(E) (1980); Phys. Lett. B 161, 141 (1985); Nucl. Phys. B 264, 99 (1986); K. S. Babu, Phys. Lett. B 203, 132 (1988); J. Garayoa and T. Schwetz, JHEP 0803, 009 (2008) [arXiv:0712.1453 [hep-ph]].

3. C.-S. Chen, C.-Q. Geng, and J. N. Ng, Phys. Rev. D 75, 053004 (2007); C.-S. Chen, C.-Q. Geng, J. N. Ng, and J. M. S. Wu, J. High Energy Phys. 0708, 022 (2007); C.-S. Chen, C.-Q. Geng, and D. V. Zhuridov, Phys. Lett. B 666340 (2008); Eur. Phys. J. C 60, 119 (2009); arXiv:0806.2698 [hep-ph].

4. P. Vogel, hep-ph/0611243; arXiv:0807.1559 [hepph]; S. M. Bilenky, arXiv:1001.1946 [hep-ph]; F. Šimkovic, Prog. Part. Nucl. Phys. 64, 219 (2010).
5. Ya. B. Zel'dovich and M. Yu. Khlopov, Pis'ma Zh. Éksp. Teor. Fiz. 34, 148 (1981) [JETP Lett. 34, 141 (1981)]; Usp. Fiz. Nauk 135, 45 (1981) [Sov. Phys. Usp. 24, 755 (1981)].

6. M. G. Shchepkin, Usp. Fiz. Nauk 143, 513 (1984) [Sov. Phys. Usp. 27, 555 (1984)].

7. M. Doi, T. Kotani, and E. Takasugi, Prog. Theor. Phys. Suppl. 83, 1 (1985).

8. R. N. Mohapatra, Phys. Rev. D 34, 3457 (1986).

9. J. D. Vergados, Phys. Lett. B 184, 55 (1987).

10. M. Hirsch, H. V. Klapdor-Kleingrothaus, and S. G. Kovalenko, Phys. Rev. Lett. 75, 17 (1995); Phys. Lett. B 352, 1 (1995); Phys. Lett. B 403, 291 (1997); Phys. Rev. D 57, 1947 (1998); M. Hirsch and H. V. Klapdor-Kleingrothaus, Nucl. Phys. B Proc. Suppl. A 52, 257 (1997).

11. K. S. Babu and R. N. Mohapatra, Phys. Rev. Lett. 75, 2276 (1995).

12. M. Hirsch, H. V. Klapdor-Kleingrothaus, and S. G. Kovalenko, Phys. Lett. B 372, 181 (1996); Phys. Lett. B 381, 488(E) (1996); H. Päs, M. Hirsch, and H. V. Klapdor-Kleingrothaus, Phys. Lett. B 459, 450 (1999).

13. A. Faessler, S. G. Kovalenko, F. Šimkovic, and J. Šchwieger, Phys. Rev. Lett. 78, 183 (1997).

14. H. V. Klapdor-Kleingrothaus and U. Sarkar, Phys. Lett. B 554, 45 (2003).

15. M. Hirsch, H. V. Klapdor-Kleingrothaus, and S. G. Kovalenko, Phys. Rev. D 54, 4207 (1996).

16. M. Hirsch, H. V. Klapdor-Kleingrothaus, and O. Panella, Phys. Lett. B 374, 7 (1996).

17. F. Deppisch and H. Päs, Phys. Rev. Lett. 98, 232501 (2007).

18. V. M. Gehman and S. R. Elliott, J. Phys. G 34, 667 (2007).

19. G. L. Fogli, E. Lisi, and A. M. Rotunno, Phys. Rev. D 80, 015024 (2009).

20. S. M. Bilenky and J. A. Grifols, Phys. Lett. B 550, 154 (2002).

21. A. Ali, A. V. Borisov, and D. V. Zhuridov, hepph/0606072; A. Ali, A. V. Borisov, and D. V. Zhuridov, Yad. Fiz. 70, 1305 (2007) [Phys. At. Nucl. 70, $1264(2007)]$.

22. A. Ali, A. V. Borisov, and D. V. Zhuridov, Phys. Rev. D 76, 093009 (2007) [arXiv:0706.4165 [hep-ph]].

23. B. M. Pontekorvo, Zh. Éksp. Teor. Fiz. 33, 549 (1957) [Sov. Phys. JETP 6, 429 (1957)]; Zh. Éksp. Teor. Fiz. 34, 247 (1958) [Sov. Phys. JETP 7, 172 (1958)]; Z. Maki, M. Nakagawa, and S. Sakata, Prog. Theor. Phys. 28, 870 (1962).

24. K. Nakamura et al. (Particle Data Group), J. Phys. G 37, 075021 (2010).

25. G. Pantis, F. Šimkovic, J. D. Vergados, and A. Faessler, Phys. Rev. C 53, 695 (1996). 
26. K. Zuber, in Proc. of the IPPP Workshop on Matrix Elements for Neutrinoless Double Beta Decay (Durham, England, 2005), Report No. IPPP-05-56, DCPT-05-114 [nucl-ex/0511009].

27. F. Šimkovic, G. Pantis, J. D. Vergados, and A. Faessler, Phys. Rev. C 60, 055502 (1999).

28. V. A. Rodin, A. Faessler, F. Šimkovic, and P. Vogel, Nucl. Phys. A 766, 107 (2006); Nucl. Phys. A 793, 213(E) (2007).

29. Majorana Collab. (C. E. Aalseth et al.), Nucl. Phys. B Proc. Suppl. 138, 217 (2005).

30. D. Budjáš, M. B. Heider, O. Chkvorets, et al., JINST 4, P10007 (2009) [arXiv:0909.4044 [nucl-ex]].

31. A. S. Barabash, Yad. Fiz. 73, 166 (2010) [Phys. At. Nucl. 73, 162 (2010)]; arXiv:0807.2948 [hep-ex].

32. I. Nasteva, PoS (EPS-HEP), 463 (2009) [arXiv:0909.3167 [hep-ex]].

33. MOON Collab. (H. Ejiri et al.), http://ewiserver. npl.washington.edu/moon/MOON-5-05.pdf;

H. Nakamura et al., J. Phys. Conf. Ser. 39, 350 (2006).
34. L. M. Ejzak, arXiv:0910.2994 [nucl-ex].

35. N. Ackerman, arXiv:0909.1826 [hep-ex].

36. V. Rodin, Prog. Part. Nucl. Phys. 64, 238 (2010).

37. J. C. Pati and A. Salam, Phys. Rev. D 10, 275 (1974); R. N. Mohapatra and J. C. Pati, Phys. Rev. D 11, 566 (1975); G. Senjanovic and R. N. Mohapatra, Phys. Rev. D 12, 1502 (1975); R. N. Mohapatra and G. Senjanovic, Phys. Rev. D 23, 165 (1981).

38. A. Faessler, G. L. Fogli, E. Lisi, et al., Phys. Rev. D 79, 053001 (2009).

39. A. S. Barabash (and the NEMO Collab.), Nucl. Phys. B Proc. Suppl. 138, 207 (2005); J. Phys. Conf. Ser. 173, 012008 (2009) [arXiv:0807.2336 [nucl-ex]]; NEMO Collab. (R. Arnold et al.), Phys. Rev. Lett. 95, 182302 (2005); Nucl. Phys. A 765, 483 (2006).

40. SuperNEMO Collab. (R. Arnold et al.), arXiv: 1005.1241 [hep-ex].

41. M. Nomachi et al., Nucl. Phys. B Proc. Suppl. 138, 221 (2005).

42. D. Akimov et al., Nucl. Phys. B Proc. Suppl. 138, 224 (2005). 